\title{
Integração ensino-serviço-comunidade na percepção de docentes de graduação em
}

\section{Enfermagem}

\author{
Teaching-service-community integration in the perception of Nursing program professors \\ Integración enseñanza-servicio-comunidad en la percepción de los profesores de la carrera de \\ Enfermeira
}

Recebido: 18/10/2021 | Revisado: 26/10/2021 | Aceito: 02/11/2021 | Publicado: 04/11/2021

\author{
Bruna da Costa Bueno \\ ORCID: https://orcid.org/0000-0001-6015-3285 \\ Universidade Federal do Paraná, Brasil \\ E-mail: bueno.brunacosta@gmail.com \\ Daiana Kloh Khalaf \\ ORCID: https://orcid.org/0000-0001-5770-7523 \\ Universidade Federal do Paraná, Brasil \\ E-mail: daianakloh@ufpr.br \\ Juliana Vieira de Moraes \\ ORCID: https://orcid.org/0000-0002-1777-7313 \\ Universidade Federal do Paraná, Brasil \\ E-mail: vieiramoraes@ufpr.br \\ Amanda Thaís Lima \\ ORCID: https://orcid.org/0000-0003-4787-9369 \\ Universidade Federal do Paraná, Brasil \\ E-mail: amanda.lima@ufpr.br \\ Carine Vendruscolo \\ ORCID: https://orcid.org/0000-0002-5163-4789 \\ Universidade do Estado de Santa Catarina, Brasil \\ E-mail: carine.vendruscolo@udesc.br \\ Edlamar Kátia Adamy \\ ORCID: https://orcid.org/0000-0002-8490-0334 \\ Universidade do Estado de Santa Catarina, Brasil \\ E-mail: edlamar.adamy@udesc.br
}

\begin{abstract}
Resumo
A integração ensino-serviço na área da saúde, neste caso, na enfermagem, proporciona aos profissionais uma prática crítica e reflexiva, sob a ótica de troca de conhecimentos entre os protagonistas. Diante disso, o objetivo deste trabalho foi identificar como ocorre a integração ensino-serviço no curso de graduação em enfermagem na percepção dos docentes. Pesquisa qualitativa do tipo descritivo-exploratória. Coleta realizada a partir de entrevistas semiestruturadas. Análise dos dados alcançada pelo referencial metodológico da Análise de Conteúdo de Bardin. Identificaram-se três categorias: 1) docentes e a integração ensino-serviço; 2) intersecção do ensino, serviço e a comunidade; e 3) relevância da integração ensino-serviço no pensamento docente. Os resultados indicam a necessidade de articulação entre os protagonistas do processo de integração ensino-serviço, os docentes compreendem a integração como um coletivo que necessita de todos os integrantes para uma prática em saúde efetiva e significativa. Sugere-se a realização de pesquisas em distintos espaços e contextos, como percepções de usuários do sistema, de preceptores, de estudantes de enfermagem e de diferentes áreas da saúde.
\end{abstract}

Palavras-chave: Educação em Enfermagem; Integração docente-assistencial; Prática do docente de Enfermagem; Ensino.

\begin{abstract}
The teaching-service integration in the health area, in this case, in nursing, provides professionals with a critical and reflective practice, from the perspective of exchanging knowledge between the protagonists. Therefore, the objective of this work was to identify how the teaching-service integration occurs in the undergraduate nursing course from the professors' perception. Descriptive-exploratory qualitative research. Data collection carried out from semi-structured interviews. Analysis through Bardin's Content Analysis. Three categories were identified: 1) professors and teachingservice integration; 2) intersection of teaching, service and the community; and 3) relevance of teaching-service integration from the professors' perspective. The results indicate the need for articulation between the protagonists of the teaching-service integration process, professors understand integration as a collective that needs all members for an
\end{abstract}


effective and meaningful health practice. Another line of research worth pursuing further is to study different spaces and contexts, such as perceptions of the health system users, tutors, nursing students and different areas of the medical field.

Keywords: Teaching care integration services; Nursing education; Nursing faculty practice; Teaching.

\section{Resumen}

La integración enseñanza-servicio en el área de la salud, en este caso, en enfermería, brinda a los profesionales una práctica crítica y reflexiva, desde la perspectiva del intercambio de conocimientos entre los protagonistas. Por tanto, el objetivo de este trabajo fue identificar cómo se da la integración enseñanza-servicio en la carrera de licenciatura en enfermería desde la percepción de los profesores. Investigación cualitativa descriptiva-exploratoria. Recolección de datos realizada a partir de entrevistas semiestructuradas. Análisis a través del análisis de contenido de Bardin. Se identificaron tres categorías: 1) profesorado e integración docente-servicio; 2) intersección de la enseñanza, el servicio y la comunidad; y 3) relevancia de la integración docencia-servicio desde la perspectiva del profesorado. Los resultados indican la necesidad de articulación entre los protagonistas del proceso de integración enseñanza-servicio, los profesores entienden la integración como un colectivo que necesita de todos los integrantes para una práctica de salud efectiva y significativa. Otra línea de investigación que vale la pena profundizar es el estudio de diferentes espacios y contextos, como las percepciones de los usuarios del sistema de salud, tutores, estudiantes de enfermería y diferentes áreas del campo médico.

Palabras clave: Servicios de integración docente asistencial; Educación en Enfermería; Práctica del docente de Enfermería; Enseñanza.

\section{Introdução}

O processo de formação dos estudantes de enfermagem é um tema amplamente discutido pela academia, tendo como uma das finalidades, superar o modelo biomédico e hospitalocêntrico, que se apresenta, atualmente, como insuficiente para o ensino de profissionais de saúde e no atendimento às necessidades dos usuários. Há urgência por uma mudança paradigmática e que se fundamenta no processo de ensino aprendizado crítico, criativo e reflexivo (Schön, 2000).

Um dos desafios do ensino em Enfermagem é a formação de profissionais da saúde competentes diante das situações reais, aquelas vividas pelos serviços de saúde. Neste ínterim, a integração entre ensino, serviço e comunidade é essencial para o crescimento do estudante, pois a sua inserção na comunidade e a prática constante de correlacionar o conteúdo teórico, ministrado em sala de aula, com a realidade dos serviços de saúde amplia o seu olhar sobre o atendimento e a contribuição do enfermeiro neste espaço (Khalaf et al., 2019; Devlin \& Duggan, 2020).

Essa aproximação entre as instituições de ensino e saúde ocorre por meio da integração ensino-serviço (IES), que é entendida como um trabalho coletivo, de pactuação e colaboração entre estudantes, docentes dos cursos de formação em saúde com os trabalhadores dos serviços de saúde e a comunidade, incluindo nessa equação todos aqueles que participam da formação do futuro profissional, de forma direta ou indireta (Albuquerque, Gomes, Rezende, Sampaio, Dias., e Lugarinho, 2008).

Destaca-se que esse processo de IES deve ser o elemento central na estratégia de elaboração curricular, sendo o ponto primordial no processo formativo dos profissionais da saúde. Um fator importante, contudo, é o alinhamento de objetivos, entre educação e saúde, como áreas do conhecimento e setores sociais, necessitam formular um novo e único campo (Zarpelon, Terencio, e Batista, 2018). A IES requer atenção ao atendimento das necessidades sociais e na capacidade de promover o desenvolvimento locorregional.

Um estudo realizado na Escola de Saúde Pública da Universidade de Washington (Mackenzie, Hinchey, \& Cornforthet, 2019) identificou que o processo de aprendizagem do estudante dentro da comunidade fortalece e consolida no mesmo um senso de responsabilidade, pois ao exercer sua prática consegue analisar e desafiar as estruturas e sistemas da comunidade, auxiliando no atendimento das necessidades da mesma.

Acredita-se que os setores de educação e saúde possuem a responsabilidade de efetivar a IES como modelo de ensinoaprendizagem, tendo em vista a centralidade do cuidado pautado no paciente e a integração entre os protagonistas. Para Beaman, 
Asano, Sibbritt, Newton, \& Davidson (2018), a tipologia da integração, pontua que todos os protagonistas possuem relevância de igual valor no que concerne ao alcance dos objetivos.

Nessa perspectiva, a potencialidade da IES no processo de formação está vinculada a ideia de participar do serviço com o objetivo de desenvolver a técnica, ainda que a mesma tenha seu grau de importância, é aproveitar infimamente o processo de ensino aprendizagem que a IES propõe, limitando o crescimento de ambos setores e com uma prática carente de uma relação dialógica (Khalaf et al., 2019).

Estudos mostram a necessidade de ressignificar a prática docente e o processo de ensino aprendizagem dos estudantes de enfermagem (Dupin et al., 2020; Gómez, Becerril, Oyola, Pineda, \& Días, 2019; Alexandre, Werneck, Chainça, \& Cesarino, 2018), mas o olhar sobre a questão não aborda a IES, a importância da inserção na comunidade antes, durante e depois da formação do profissional de enfermagem, além de, principalmente, a prática docente sob a perspectiva do interperíodo e do multiprofissional e, quando citados são solutos, sem a percepção de que uma prática efetiva necessita da conjunção de todos esses elementos.

Discutir e explorar o processo de formação e de ensino-aprendizagem é de corresponsabilidade de ambos os setores, dessa forma, há de se pretender uma retroalimentação nos padrões de parceria intersetorial, em que o objetivo final seja a formação de um profissional preparado para o mercado de trabalho. Neste estudo, objetivou-se identificar como ocorre a IES no curso de graduação em enfermagem na percepção dos docentes

\section{Metodologia}

Foram utilizados os princípios da metodologia qualitativa descritivo-exploratória. A abordagem qualitativa enquadrase neste estudo ao proporcionar o aprofundamento da investigação das questões relacionadas ao fenômeno em estudo e suas relações, mantendo aberta a percepção da individualidade e os significados múltiplos. Ressalta-se que as pesquisas de caráter exploratório tem por objetivo principal desenvolver, esclarecer e modificar conceitos e ideias sobre um tema a ser investigado, enquanto a pesquisa descritiva tem a finalidade de descrever as características de determinada população ou fenômeno (Gil, 2011; Polit \& Beck, 2011).

Inicialmente realizou-se duas entrevistas piloto para aprimorar o instrumento norteador da pesquisa. Foram entrevistados para o estudo 10 enfermeiros docentes, com atuação superior a dois anos no curso de graduação em enfermagem, de uma universidade pública localizada na Região Sul do Brasil. Os participantes atuam na Atenção Primária em Saúde.

A coleta de dados foi realizada a partir de entrevistas individuais com roteiro semiestruturado aplicadas aos docentes entre dezembro de 2019 e junho de 2020, com duração de aproximadamente 30 minutos, em sala privada e silenciosa ou por meio de chamadas de vídeo, ambas agendadas em hora e data convenientes para o docente. O roteiro é oriundo de um estudo documental realizado previamente pelas pesquisadoras, cujas questões são frutos de pesquisas anteriores realizadas em outras instituições com o intuito de avaliar o processo de IES.

A análise dos dados foi alcançada pelo referencial metodológico da Análise de Conteúdo de Bardin, na modalidade Análise Categorial Temática, um conjunto de técnicas de análise de comunicação capaz de formar indicadores que permitam tornar replicáveis e válidas inferências sobre dados de determinado contexto. A saber divide-se em três etapas: pré-análise; exploração do material; e tratamento dos dados (Bardin, 2016).

Inicialmente, foi realizada a leitura flutuante de todo material coletado para dimensionar as falas e organização das transcrições mediante o uso do software webQDA (Web Qualitative Data Analysis), no qual construíram-se em códigos árvores e códigos livres, que em conjunto estruturam as categorias, representando a codificação dos dados e a definição das categorias. 
Por fim, o tratamento dos dados deu-se pela reflexão, articulação e discussão dos resultados obtidos com a literatura (Bardin, 2016).

Para resguardar a identidade dos entrevistados, optou-se pela utilização de códigos de identificação dos docentes. Tais códigos foram iniciados pela letra $\mathrm{P}$, de participante, seguidos por um número cardinal, que significou a ordem cronológica de desenvolvimento das entrevistas.

Este estudo obteve parecer positivo do Comitê de Ética, com o Certificado de Apresentação para Apreciação Ética em Pesquisa (CAAE) sob o nº 22728219.4.0000.0102. Os docentes receberam informações orais e escritas do estudo e a participação foi estritamente voluntária, sendo informados de que poderiam desistir do estudo a qualquer momento, sem consequências para sua formação.

\section{Resultados}

Após a análise das entrevistas com os docentes identificaram-se três categorias principais relacionadas à percepção dos docentes de um curso de graduação em enfermagem a respeito da IES: docentes e a integração ensino-serviço; intersecção do ensino, serviço e a comunidade; e relevância da IES no pensamento docente.

A subcategoria docente e a integração ensino-serviço retratam o entendimento dos docentes acerca do ensino na relação integração ensino-serviço com o objetivo de levantar a importância dessa interação. Os docentes optaram por falar das práticas, descrevendo a passagem dos estudantes do início das práticas até o estágio supervisionado, além do acompanhamento do profissional de enfermagem na assistência.

Os docentes reconhecem que as vivências e visitas técnicas iniciam-se nos primeiros períodos do curso de graduação em Enfermagem e as aulas práticas específicas iniciam-se no quarto período. Relacionam o início das atividades práticas, predominantemente, como sendo adequados para o processo de formação dos estudantes (80\%) ou não adequados (20\%) para o processo de ensino-aprendizagem, porém existem participantes que destacam que a IES deveria começar desde o primeiro período.

[...] Penso o seguinte, da maneira que o nosso Projeto Político Pedagógico, nosso processo de ensino está colocado, o $4^{\circ}$ período é interessante [...]. (P6)

[...] Considero que as atividades práticas deveriam iniciar já no primeiro período do curso [...]. (P9)

Dentre os depoimentos, observa-se uma congruência em entender a importância do alinhamento das atividades com o projeto pedagógico proposto, estabelecendo-se objetivos que buscam conversar com as necessidades do serviço e com aqueles que almejam alcançar com os estudantes ao longo do semestre, afirmando-se o valor do conhecimento e vínculo com os profissionais, desde enfermeiros e autoridade sanitária até os demais servidores.

[...] primeiro o alinhamento entre os professores da disciplina, qual é o objetivo dessa atividade, porquê que a gente está levando o aluno para campo e o que a gente quer que o aluno desenvolva, depois a gente precisa, a partir desse planejamento, identificar qual é o cenário, o campo que vai permitir que o aluno desenvolva isso. (Pl)

Alguns relatos identificam-se ao estabelecer o processo de avaliação como construtivo e dialógico. Apresenta-se o conhecimento como progressivo e, portanto, é considerada a autoavaliação, bem como a avaliação do docente, da disciplina e do supervisor, profissional da enfermagem que acompanha o estudante no último período, durante estágio supervisionado. 
[...] Autoavaliação dos alunos, avaliação pelos profissionais que o acompanharam o discente durante o estágio e pelo docente. (P5)

A subcategoria intersecção do ensino, serviço e comunidade está relacionado com a participação da comunidade no autocuidado, as barreiras na receptividade da instituição na unidade. Os docentes apontaram três aspectos centrais em suas falas: comunidade como espectadora do cuidado, alunos do último ano estão mais preparados para a prática em saúde e recusa no atendimento das consultas de enfermagem.

A participação da comunidade no processo da IES destaca-se por divergir nos depoimentos dos docentes. Em algumas falas, apresenta-se a participação da comunidade como termômetro do cuidado realizado pelo estudante e pelo docente que está supervisionando, enquanto outras destacam a necessidade da articulação dos estudantes e docentes com os representantes da comunidade.

[...] Na verdade até falta, eu entendo que o correto seria a gente também articular com a associação de moradores, explicar o que os alunos vão fazer, pedir para eles opinarem, dizer o que eles acham, o que a gente poderia contribuir etc e tal, na verdade isso não acontece, a comunidade acaba sendo uma espectadora e recebe aquilo que a gente acha que deve ser feito pelos alunos, essa relação está aquém do desejado. (P8)

Os docentes sinalizam a boa receptividade dos usuários do serviço ao cuidado realizado pelo estudante, sendo enfatizada a supervisão do docente como auxiliadora no processo de receptividade. Em contrapartida, foram relatadas algumas situações em que o usuário não recebeu a equipe, tanto estudante quanto docente, ao julgar que receberia atendimento médico.

[...] De modo geral, recebem bem as atividades desenvolvidas pelos discentes, como são alunos mais avançados no curso, os próprios alunos têm mais segurança e passam isso para os usuários, o que contribui para a aceitação. (P5) [...] alguns usuários se recusam de fazer alguns procedimentos [...](P1)

Os docentes relatam variados elementos que estruturam de que forma se dá a receptividade dos profissionais. Comumente, a receptividade é adequada, proporcionando momentos de capacitação dos profissionais por meio da troca de saberes com os estudantes, bem como pelo vínculo dos profissionais com os docentes. A carência estrutural do serviço, a maneira como as equipes multiprofissionais estão organizadas e, por vezes, o momento político, interferem numa boa receptividade do campo de prática.

[...] [A receptividade é] boa, na grande maioria entendem esta parceria. Quando ocorre resistência, uma das causas é devido ao excesso de atividades sob responsabilidade do profissional (dimensionamento equivocado nos serviços de saúde), mas são raras as vezes onde temos essa receptividade. (P5)

A subcategoria relevância da IES no pensamento docente retrata os aspectos positivos da integração entre o meio acadêmico e a prática, comentando sobre o envolvimento direto do município, servidores da unidade, instituição acadêmica, docentes e alunos. Além de demonstrar as vantagens na integração do aluno com a realidade dos serviços do Sistema Único de Saúde (SUS).

Ao indicar os participantes do processo de IES, os depoimentos convergem para o entendimento de que os docentes, os estudantes, os profissionais do campo, as instituições prestadoras do serviço, a gestão e demais atores envolvidos no processo direto ou indireto da aprendizagem do estudante são constituintes da integração. 
[...] Acho que todos os atores que estão envolvidos, desde a coordenação do curso, os professores da disciplina, os alunos, a gestão dos serviços de saúde, a secretaria de saúde, enfim, os profissionais do serviço, o enfermeiro que é a nossa ligação direta, mas todos os profissionais e os usuários do serviço. (P1)

A IES é entendida pelos docentes como essencial para todos os protagonistas desse processo, enfatizando a sua relevância como meio que oportuniza ao estudante desenvolver suas habilidades no cuidado ao vivenciar a realidade. Abordase, inclusive, a educação como uma prática social e problematizadora para o desenvolvimento de sujeitos sociais críticos e reflexivos.

[...] a integração ensino-serviço para a enfermagem, ela é essencial porque o serviço precisa se aproximar do processo de formação do aluno e o outro lado também é verdade, a academia precisa se aproximar do serviço, não tem condição da academia viver num mundo em que não é a realidade. (P6)

\section{Discussão}

A integralidade do cuidado é concebida como o princípio fundamental das práticas em saúde, determinando uma forma de cuidar que ultrapassa o modelo biomédico e centraliza o cuidado em prol dos usuários do serviço e da comunidade na qual está inserido. Isto é, entendendo-os como seres sociais e históricos, que necessitam de uma assistência que os compreenda na plenitude de suas necessidades e não apenas no saber fazer mecanizado (Silveira J, Kremer, Silveira M, e Schneider, 2020).

Observa-se um esforço dos programas de educação em enfermagem em afastar-se do modelo tradicional de ensino, da educação bancária $^{1 *}$ e rígida, que impede o estudante de atuar com qualidade. No lugar de tais práticas, percebe-se uma busca em preparar os estudantes para o seu papel social, de práticas interconectadas e reflexivas tendo por objetivo desenvolver nos mesmos um pensamento crítico e reflexivo sobre a sua prática (Tsimane e Downing, 2020).

Todavia, a formação em enfermagem ainda se apresenta como um grande desafio apresentado pelas docentes, considerando a configuração dos nossos currículos que ainda mantém um caráter de lógica flexneriana, a sua carência em se adequar a realidade do meio social, político e cultural, a qualidade questionável do mesmo ao predominar o conteúdo teórico em detrimento das práticas e vivências nas estruturas sanitárias, entre outros aspectos que distanciam o estudante do concreto e de uma prática profissional com qualidade (Ximenes et al., 2020), configurando uma realidade que se estende pelo país e por diferentes cursos da área da saúde, como apresentado por Oliveira, Amaral, Cyrino, e Gianini (2021), que aponta a contínua transferência de conhecimentos na processos de ensino aprendizagem baseados em desenvolvimento de habilidades como superados, expondo a necessidade do pensamento crítico-reflexivo de uma perspectiva histórico-dialética.

Proporcionar aos estudantes diferentes cenários de ensino apresenta-se como estratégia colocada pelos docentes como fundamental na formação, assim como sustentado no estudo de Rodriguez (2021), transpondo as limitações estruturais de salas de aula e inserindo os alunos nos mais diversificados cenários de prática, para identificar e desenvolver estratégias que atendam as reais necessidades dos serviços, das famílias, pessoas e comunidades, concedendo vivências de realidades próximas e as dificuldades do sistema de saúde, permitindo reconhecer as comunidades que, posteriormente, podem vir a ser seu território de trabalho (Lima et al., 2019).

A própria legislação brasileira corrobora afirmando que, ao integrar-se ao mundo do trabalho, o mundo da educação faz com que o aprendizado seja fundamentado na reflexão das práticas, ganhando sentido por estar relacionado à realidade do trabalho em saúde. Deste modo, recomenda-se que os estudantes devem ser inseridos nos cenários de prática desde o início da formação, rompendo com a dicotomia teoria-prática (Brasil, 2005; Brasil, 2017).

$1^{*}$ Em Introdução à Psicologia Escolar. São Paulo: Casa do Psicólogo, 1997 (3ª edição). Capítulo V, p.61-69 
No momento de planejar as atividades em campo, os docentes contam com o conhecimento da realidade do serviço e dos vínculos formados entre a academia e os servidores, buscando alinhar os objetivos que almejam alcançar com os estudantes ao longo do semestre. Esse alinhamento promove um objetivo em conjunto, permitindo que a atuação do ensino sob o serviço seja feita de acordo com a realidade do território da comunidade.

As atividades são elaboradas pelos docentes em parcerias, que compartilham entre si conhecimentos e estratégias que advém da experiência profissional de cada um, contribuindo para um planejamento fundamentado e revisado por vários olhares. Além disso, são formuladas tendo em vista o currículo vigente e as possibilidades de execução, muitas vezes limitadas pela configuração das instituições envolvidas, seja universidade ou serviço de saúde. Outros fatores a serem considerados são a dificuldade de acesso aos serviços e a carência de docentes ao número de estudantes matriculados, que não permitem a todos os envolvidos um aproveitamento da prática em campo na sua plenitude (Lima et al., 2019; White, 2017).

A avaliação dos estudantes, via de regra, ocorre de modo processual e dialógico através de avaliação por competências, por meio de instrumentos. Os depoimentos analisados abordam a participação dos estudantes no processo de avaliação e as contribuições dos servidores nesse processo, ao compor a avaliação final. O progresso do estudante é avaliado então a partir de vários olhares, professor, servidor e o do mesmo.

Neste seguimento, torna-se imprescindível debater a ideia de avaliação processual e dialógica. Deve-se partir da alegação de uma avaliação formativa e não classificatória, comumente utilizada pela educação bancária e que não demonstra o crescimento do estudante de modo efetivo (Ribeiro, Veloso e Zanardiet, 2020).

Sinalizando o aprender como um processo de construção do conhecimento, no contexto em que a educação em enfermagem está situada, a dinâmica entre o docente com o papel de facilitador e o engajamento do estudante em aprender promove uma sinergia para a conquista de uma aprendizagem transformadora (Tsimane e Downing, 2020).

Ao estabelecer a relação entre o ensino, o serviço e a comunidade, os depoimentos expõem a participação da comunidade como satisfatória, a boa receptividade alicerçada pelo vínculo estabelecido entre o docente e o serviço, a segurança transmitida pelo estudante juntamente com a supervisão no cuidado prestado. Em contrapartida, fica perceptível que alguns usuários não recebem o atendimento por parte do estudante e docente, confirmando uma herança histórica e social, da consulta de enfermagem como inferior à consulta médica, mas reiteram que esta não é uma prática habitual em campo.

A receptividade dos profissionais da saúde, sob a perspectiva dos entrevistados, tende a ser agradável. Quando esta não ocorre, frequentemente relaciona-se a pouca receptividade com a falta de recursos materiais, equipamentos, estrutura física do serviço e a sobrecarga de atividades devido à alta demanda de pacientes que, associada à falta de recursos humanos, tem sido uma constante, aliada ao sentimento de falta de reconhecimento da sociedade sob o seu trabalho, que gera sentimentos de ansiedade, estresse e frustrações no profissional (Marques et al., 2020).

A troca de conhecimentos nos setores da educação e saúde é de grande valia, a experiência profissional, da prática do dia a dia contribui grandemente para a formação do estudante. A sua constante atualização, pautada nas principais evidências científicas, contribui para o processo de educação continuada dos profissionais do serviço que, muitas vezes, pela forma como a sua ocupação está organizada, não consegue dar continuidade nos estudos. O processo de mentoria é apontado por estudos como essencial para o processo de formação dos estudantes e para a continuidade dos profissionais da ponta, pois, ao auxiliar os estudantes no desenvolvimento de suas habilidades, o mentor analisa e fortalece seus conhecimentos (Hafsteinsdóttir, Schoonhoven, Hamers, \& Shuurmans, 2020). Com esta integração, as práticas tornam-se críticas, contribuindo de modo significativo na formação de profissionais aptos a identificar, planejar e intervir sobre os reais problemas de saúde (Nogueira et al., 2019). 
De acordo com os depoimentos, os participantes do processo de IES são todos os envolvidos na formação dos estudantes e das instituições de saúde, entendendo a integração como o produto de uma ação coletiva, que engloba os estudantes, docentes e as devidas coordenações do Sistema de Saúde (Khalaf et al., 2019). Em contrapartida, dificilmente menciona-se a comunidade como protagonista desse processo, firmando a questão da dificuldade de se estabelecer vínculos significativos com os usuários, capazes de transformar a realidade, bem como o cuidado, às ações e estratégias acabam em ocorrendo de modo vertical, do olhar do enfermeiro, estudante ou docente sob as necessidades em saúde do usuário e da comunidade (Dupin et al., 2020).

A partir de alguns depoimentos, entende-se a concepção de IES pela percepção dos docentes como essencial para a aproximação entre o ensino e o serviço, ao manifestar a integração como a prática social da educação que pressupõe reconhecer a relação dinâmica das partes entre si e com o todo, bem como ao proporcionar a compreensão dos fenômenos estruturais que determinam o processo de saúde e de educação. A sua importância parte da premissa da troca de informações entre ensino e serviço, permitindo ao estudante desfragmentar seu conhecimento através da correlação com a prática, compromisso entre a saúde e educação (Nogueira et al, 2019), salientando que essas trocas são como vias de mão dupla que acrescentam em ambos os sujeitos, de forma que o estudante traga essa visão atualizada sobre os processos de saúde e doença e que o profissional enfrente a realidade do ser enfermeiro.

\section{Considerações Finais}

Os resultados indicam a importância da integração em todos os âmbitos envolvidos, sendo eles o ensino enquanto academia, estudantes e docentes; os serviços de saúde, enquanto profissionais da enfermagem em campo de trabalho; e a comunidade, protagonista do cuidado, como ser social e histórico, carente de uma assistência que a contemple em sua plenitude. Para isso, é necessário fortalecer políticas públicas para ações que permitam uma maior aproximação com a realidade do território, e com as práticas colaborativas e interprofissionais. Chama-se a atenção para a necessidade de pensar a integração ensino serviço e sua articulação com a comunidade.

A partir do estudo realizado, é possível afirmar que é necessário explorar e fomentar a discussão sobre a IES. Especial destaque para pesquisas que explorem a participação da comunidade na IES e os impactos do mundo moderno nas oportunidades de sua participação na centralidade do cuidado.

Algumas considerações sobre os resultados desta pesquisa podem ser identificadas como limitadas, por exemplo, a dificuldade de ampliar a amostra docente e desvelar maior riqueza de conteúdo ou diversidade de percepção, pois responderam à entrevista os docentes que se dispuseram a fazê-lo, o que, por si só, poderia representar um viés. Convidamos os docentes que tinham atividades na atenção básica de saúde. Essa adesão se alinha à observação da sobrecarga de atividades dos professores e da falta de profissionais para suprir a demanda que a academia necessita. Não obstante, o estudo fornece pistas e indagações àqueles que dirigem as iniciativas de integração ensino-serviço-comunidade, sejam elas relativas à realidade da academia, o processo de planejamento e avaliativo, e a importância da IES pelos docentes. Ainda se mostraram como processos importantes a estrutura organizacional, o ambiente cooperativo dos docentes, a complexidade da inovação frente às instituições e a própria estrutura curricular, a capacitação docente, a comunicação e estabelecimento de vínculos com preceptores.

Por fim, é necessário refletir sobre a necessidade de continuidade da pesquisa sobre a integração ensino-serviçocomunidade. Sugerem-se pesquisas que fortaleçam o diálogo com o setor de saúde e o setor de educação, estabelecendo um apoio a preceptoria docente, a atuação prática dos estudantes e a participação da comunidade, para compreender a integração ensino-serviço por diferentes perspectivas, e para delimitar as ações frente às necessidades que se apresentam na prática. 


\section{Referências}

Albuquerque, V. S., Gomes, A. P., Rezende, C. H. A., Sampaio, M. X., Dias O. V., \& Lugarinho, R. M. (2008). A Integração Ensino-serviço no Contexto dos Processos de Mudança na Formação Superior dos Profissionais da Saúde. Rev Bras Edu Médica, 32 (3): 356 - 362. https://www.scielo.br/j/rbem/a/YSfdZCkkTd9KSvd8Vjmhsqn/abstract/?lang=pt

Alexandre, K. C. R. S., Werneck, A. L., Chainça, E., \& Cesarino, C. B. (2018). Docência em cursos superiores de enfermagem: formação e práticas pedagógicas. Rev baiana enferm, 32:e24975. DOI: 10.18471/rbe.v32.24975.

Bardin, L. (2016). Análise de Conteúdo. São Paulo: Edições 70.

Beaman, A., Asano, R., Sibbritt D., Newton, P.J., \& Davidson, P.M. (2018). Global service learning and health systems strengthening: An integrative literature review. Heliyon, [S.1.] v. 4, e00713. doi: 10.1016/ j.heliyon.2018.e00713

Brasil. (2017). Conselho Nacional de Saúde (CNS). Resolução $n^{o} 569$ de 8 de dezembro de 2017. http://conselho.saude.gov.br/resolucoes/2017/Reso569.pdf

Brasil. (2005). Conselho Nacional de Saúde (CNS). Resolução no 350, de 09 de junho de 2005. https://conselho.saude.gov.br/resolucoes/2005/Reso350.pdf

Devlin, N., \& Duggan, S. (2020). An evaluation of nurses' experiences of mentoring pre-registration students. British Journal of Nursing, Vol 29, No 5. doi: 10.12968 / bjon.2020.29.5.308

Dupin, C. M., Pinon, M., Jaggi, K., Teixeira, C., Sagne, A., \& Delicado, N. et al. (2020) Public health nursing education viewed through the lens of superdiversity: a resource for global health. BMC Nursing, 19:18. https://doi.org/10.1186/s12912-020-00411-3

Gil, A.C. (2011). Métodos da pesquisa social. São Paulo: Atlas, 6. Ed.

Gómez, M. A. J, Becerril, L. C., Oyola, M. B. V., Pineda, M. C., \& Días, L. Y. B. (2019). Reflective and critical thinking in nursing curriculum. Rev. LatinoAm. Enfermagem, 27:e3173. http://dx.doi.org/10.1590/1518-8345.2861.3173.

Hafsteinsdóttir, T. B., Schoonhoven, L., Hamers, J., \& Shuurmans, M. J. (2020). Leadership Mentoring in Nursing Research. Journal of Nursing Scholarship, 52:4, 435-445. doi:10.1111/jnu.12565

Khalaf, D. K., Reibnitz, K. S., Vendruscolo, C., Lima, M. M., Oliveira, V. B. C. A., \& Correa, A. B. (2019). Integração ensino-serviço sob a percepção dos seus protagonistas. Rev. de Enfermagem da UFSM. Santa Maria, v. 9, n. 2, p. 1-20. doi: 10.5902/2179769231464.

Lima, M. A. D. S., Marques, G. Q., Damaceno, A. N., Santos, M. T., Witt, R. R., \& Acosta, A. M. (2019). Instrumentos de avaliação de estruturação de redes de cuidados primários: uma revisão integrativa. Saúde Debate. Rio de Janeiro, V. 43, n. especial 5, P. 299-311, dez. doi: 10.1590/0103-11042019S524

Mackenzie, S., Hinchey, D., \& Cornforth, K. (2019). A Public Health Service-Learning Capstone: Ideal for Students, Academia and Community. Front. Public Health. Lausanne, v.7 n. 10, p. 1-7, jan. doi: 10.3389 / fpubh.2019.00010

Marques, C. R., Ribeiro, B. M. S. S., Martins, J. T., Dias, H. G., Darli, R. C. M. B., Bernardes, M. L. G., \& Karino, M. E. (2020). Fatores de satisfações e insatisfações no trabalho de enfermeiros. Rev enferm UFPE online, 14:e244966. https://doi.org/10.5205/1981-8963.2020.244966

Neto, F. R. G. X., Neto, D. L., Cunha, I. C. K. O., Ribeiro, M. A., Freire, N. P., Kalinowski, C. E., Oliveira, E. N., \& Albuquerque, I. M. N. (2020). Reflections on Brazilian Nursing Education from the regulation of the Unified Health System. Ciência \& Saúde Coletiva, 25(1):37-46. https://doi.org/10.1590/141381232020251.27702019

Nogueira, I. S., Maldonado, R. N., Labegalini, C. M. G., Jaques, A. E., Carreira, L., \& Baldissera, V. D. A. (2019). Contribuições da integração ensino-serviçocomunidade para a formação e transformação de práticas na saúde do idoso. Enfermagem Brasil, 18(2):193-200. https://doi.org/10.33233/eb.v18i2.2077

Oliveira, C. A., Amaral, E. M., Cyrino, E. G., \& Gianini, R. J. (2021). Encontros e desencontros entre projetos pedagógicos de cursos de Medicina e diretrizes curriculares nacionais: percepções de professores. Interface (Botucatu), 25: e200076. https://doi.org/10.1590/interface.200076

Polit, D. F., \& Beck, C. T. (2011). Fundamentos de Pesquisa em Enfermagem. Porto Alegre : Artmed, 7. ed.

Rodríguez, A. M. M. M., Cardoso, T. Z., Abrahão-Curvo, P., Gerin, L., Palha, P. F., \& Segura-Muñoz, S.I. (2021). Vacinação contra influenza no enfrentamento da COVID-19: integração ensino-serviço para formação em enfermagem e saúde. Esc Anna Nery, 25(spe):e20200379 https://doi.org/10.1590/2177-9465-EAN2020-0379

Ribeiro, M. P., Veloso, S. G. A., \& Zanardi, T. A. C. (2020). Educação integral e integrada: a avaliação emancipatória a caminho de uma lógica dialógica. Revista Cocar, V.14 N.28 Jan./Abr./ p.541-563 https://periodicos.uepa.br/index.php/cocar/article/view/3137

Schön, D. Educando o profissional reflexivo: um novo design para o ensino e aprendizagem. Trad. Roberto Cataldo Costa. Porto Alegre: Artes Médicas Sul, 2000

Silveira, J. L. G. C., Kremer, M. M., Silveira, M. E. U. C., \& Schneider, A. C. T. C. (2020). Percepções da integração ensino-serviço-comunidade: contribuições para a formação e o cuidado integral em saúde. Interface (Botucatu). Botucatu, v. 24, e190499. https://doi.org/10.1590/Interface.190499

Tsimane, T. A., \& Downing, C. (2020). Transformative learning in nursing education: A concept analysis. International Journal of Nursing Sciences, Volume 7, Issue 1, 10 January 2020, Pages 91-98. https://doi.org/10.1016/j.ijnss.2019.12.006

White, E. (2017). A Comparison of Nursing Education and Workforce Planning Initiatives in the United States and England. Policy Polit Nurs Pract. November; 18(4): 173-185. doi:10.1177/1527154418759666

Zarpelon, L. F. B., Terencio, M. L., \& Batista, N. A. (2018). Integração ensino-serviço no contexto das escolas médicas brasileiras: revisão integrativa. Ciência \& Saúde Coletiva, 23(12):4241-4248. https://doi.org/10.1590/1413-812320182312.32132016 